Annals of Pure and Applied Mathematics

Vol. 16, No. 1, 2018, 69-80

ISSN: 2279-087X (P), 2279-0888(online)

Published on 1 January 2018

www.researchmathsci.org

DOI: http://dx.doi.org/10.22457/apam.v16n1a9

Annals of

Pure and Applied

Mathematics

\title{
Vertex Distance Complement Spectra of Some Graphs
}

\author{
Renny P Varghese ${ }^{1}$ and Susha $D^{2}$
}

Department of Mathematics, Catholicate College, Pathanamthitta

Kerala, India - 689645.

E-mail: ${ }^{1}$ rennypv1@gmail.com, ${ }^{2}$ sushad70@gmail.com

Received 6 December 2017; accepted 26 December 2017

Abstract. In this article we determine the $V D C$ - spectrum of some class of graphs. We mainly discuss the $V D C$ - spectrum of join of two graphs, Cartesian product, double graph, lexicographic product, double odd graph and extended double cover graph. We observe that under certain conditions some graphs in the above classification are $V D C$ integral. Also, we characterize that the Cartesian product of a graph $G$ with $K_{2}$ and the extended double cover graph of $G$ are $V D C$-equienergetic graphs.

Keywords: vertex distance complement matrix, join of graphs, Cartesian product, lexicographic product, double graph, double odd graph, extended double cover graph, $V D C$ - energy.

AMS Mathematics Subject Classification (2010): 05C12, 05C50, $05 \mathrm{C} 76$

\section{Introduction}

Let $G$ be a simple undirected graph on $n$ vertices. The adjacency matrix of $G$ denoted by $A(G)=\left(a_{i j}\right)_{n \times n}$ is an $n \times n$ symmetric matrix indexed by the vertices $\left\{v_{1}, v_{2}, \ldots, v_{n}\right\}$ of $G$ where $a_{i j}=1$ if $v_{i}$ and $v_{j}$ are adjacent in $G$ and is 0 otherwise. A graph is regular if every vertex has the same degree. The characteristic polynomial of $G$ is defined as $f_{G}(x)=\operatorname{det}\left(x I_{n}-A\right)$ where $I_{n}$ is the identity matrix of order $n$. The roots of the characteristic equation of $A$ are called the eigenvalues of $G$. It is denoted by $\lambda_{1}(G) \geq \lambda_{2}(G) \geq \cdots \geq \lambda_{n}(G)$ and are called A - spectrum of $G$.

The distance matrix $D(G)=\left(d_{i j}\right)$ where $d_{i j}=d\left(v_{i}, v_{j}\right)$ is the distance (the length of the shortest path) between the vertices $v_{i}$ and $v_{j}$. If the diameter of $G$ is atmost two, any pair of non adjacent vertices is at a distance less than or equal to two. Graham and Pollak [5] introduced the distance matrices in 1971. The matrix $D(G)$ is non negative, irreducible and symmetric, the eigenvalues of $D(G)$ are real. Let $G$ be a graph with diameter atmost 2, then $D(G)=A(G)+2 \overline{A(G)}=2(J-I)-A(\overline{G)}$ [6] where $\bar{A}$ is the adjacency matrix of the complement graph $\bar{G}$. If $\eta_{i_{1}} \geq \eta_{i_{2}} \geq \cdots \geq \eta_{i_{g}}$ are the distinct eigenvalues of $D(G)$ with corresponding algebraic multiplicity $m_{i_{1}}, m_{i_{2}}, \ldots, m_{i_{g}}$ and $m_{i_{1}}+m_{i_{2}}+\ldots+m_{i_{g}}=n$, then the $D$ - spectrum can be written as

$$
\operatorname{Spec}_{D}(G)=\left(\begin{array}{lll}
\eta_{i 1} & \eta_{i 2} & \eta_{i g} \\
m_{i 1} & m_{i 2} & m_{i g}
\end{array}\right) \text {. }
$$




\section{Renny P Varghese and Susha D.}

Now consider a special class of graphs called chemical graphs, which representing the chemical structure of a compound. Molecular graphs are graphs in which the chemical structure under consideration are molecules. Also, these molecular graphs are undirected graphs. In molecular graphs, vertices correspond to atoms and edge represents covalent bond between atoms and usually the hydrogen atoms are neglected. These matrices have been used to determine a number of topological indices like Balaban index, Winer index, distance sum index etc. There are some models for the molecular design of a chemical compound. Quantitative structure property relationship (QSPR) and quantitative structure activity relationship (QSAR) are two such models. Renny and Susha in [12] determined the $V D C$ - spectrum of the molecular matrices derived from the graph distance namely vertex distance complement matrix $(V D C)$. In this paper we find the VDC spectrum of some class of graphs namely join of two graphs, cartesian product, double graph, lexicographic product, double odd graph and extended double cover graph.

The organization of the paper is as follows. In section 2 we mention some basic results on spectral graph theory which are useful to prove the results in the succeeding sections. In section 3 we determine the $V D C$ - spectrum of some class of graphs. We also discuss about some family of graphs which are $V D C$ - integral. Then in section 4 we discuss the application of $V D C$ - spectrum such as $V D C$ - energy of $G \times K_{2}$, $G\left[K_{2}\right], \quad D_{2}(G)$ and the extended double cover graph. We conclude that $G \times K_{2}$ and extended double cover graph of $G$ are $V D C$ - equienergetic graphs.

\section{Preliminaries}

Definition 2.1. [8] The vertex distance complement matrix $V D C=V D C(G)$ of a graph $G$ with ' $n$ ' vertices is an $n \times n$ symmetric matrix $V D C=\left[\mathrm{c}_{\mathrm{ij}}\right]$, where

$d_{i j}$ is the distance between the vertices $v_{i}$ and $v_{j}$.

$$
\mathrm{c}_{\mathrm{ij}}= \begin{cases}n-d_{i j}, & \text { if } \mathrm{i} \neq \mathrm{j} \\ 0, & \text { if } i=j\end{cases}
$$

The eigenvalues of $\operatorname{VDC}(G)$ are denoted by $\theta_{1} \geq \theta_{2} \geq \cdots \geq \theta_{n}$ and are called the $V D C$ - eigenvalue of $G$. The set of all $V D C$ - eigenvalues of $G$ is called the $V D C$ - spectrum of $G$. Two non isomorphic graphs are said to be $V D C$ - cospectral if they have the same $V D C$ - spectrum. A graph is $V D C$ - integral if the $V D C$ - spectrum consists only of integers.

Definition 2.2. [3] Let $\mathrm{G}$ be a simple connected graph. $\mathrm{G}$ is called distance regular if it is regular, and if for any two vertices $u, v \in \mathrm{V}(\mathrm{G})$ at a distance $i$, there are constant number of neighbors $c_{i}$ and $b_{i}$ of $v$ at a distance $i-l$ and $i+l$ from $u$ respectively.

Theorem 2.1. [12] Let $G$ be a $r$-regular graph with $n$ vertices and $\operatorname{diam}(G)=2$. Let $\left\{r, \lambda_{2}, \ldots, \lambda_{n}\right\}$ be the adjacency eigenvalues of $G$, then $V D C$ - eigenvalues of $G$ are $(n-1)(n-2)+r$ and $\lambda_{i}-n+2$ for $i=2,3, \ldots, n$.

Lemma 2.2. [4] Let $G$ be a connected $r$ - regular graph on ' $n$ ' vertices with its adjacency matrix A having $n$ distinct eigenvalues $r=\lambda_{1}, \lambda_{2}, \ldots, \lambda_{n}$. Then there exists a polynomial $\mathrm{P}(\mathrm{x})=\mathrm{n} \frac{\left(\mathrm{x}-\lambda_{2}\right)\left(\mathrm{x}-\lambda_{3}\right) \ldots\left(\mathrm{x}-\lambda_{\mathrm{n}}\right)}{\left(\mathrm{r}-\lambda_{2}\right)\left(\mathrm{r}-\lambda_{3}\right) \ldots\left(\mathrm{r}-\lambda_{\mathrm{n}}\right)}$ such that $\mathrm{P}(\mathrm{A})=\mathrm{J}$ where $\mathrm{J}$ is the square matrix of order $n$ 


\section{Vertex Distance Complement Spectra of Some Graphs}

whose all entries are one, so that $\mathrm{P}(r)=n$ and $\mathrm{P}\left(\lambda_{i}\right)=0$ for all $\lambda_{i} \neq r$.

Lemma 2.3. [4] Let $G$ be a connected $r$ - regular graph with adjacency matrix A and spectrum $\left\{r=\lambda_{1}, \lambda_{2}, \ldots, \lambda_{n}\right\}$. Then the adjacency matrix and spectrum of $\bar{G}$, the complement of the graph $G$, are $\bar{A}=\mathrm{J}-\mathrm{I}-\mathrm{A}$ and $\left\{n-r-1,-\left(\lambda_{2}+1\right), \ldots,-\left(\lambda_{n}+1\right)\right\}$ respectively. Here $\mathrm{J}$ denote matrix with all entries equal to one and I denote the unit matrix.

Lemma 2.4.[4] Let $\mathrm{A}=\left[\begin{array}{ll}M_{1} & M_{2} \\ M_{2} & M_{1}\end{array}\right]$ be a $2 \times 2$ block symmetric matrix. Then the eigenvalues of $A$ are that of $M_{1}+M_{2}$ together with $M_{1}-M_{2}$.

Theorem 2.5. [6] Let $\mathrm{D}$ be the distance matrix of a connected regular graph $\mathrm{G}$ on ' $n$ ' vertices with its distinct eigenvalues $k=\eta_{1}, \eta_{2}, \ldots, \eta_{n}$. Then there exists a polynomial $P(x)=n \frac{\left(x-\eta_{2}\right)\left(x-\eta_{3}\right) \ldots\left(x-\eta_{n}\right)}{\left(k-\eta_{2}\right)\left(k-\eta_{3}\right) \ldots\left(k-\eta_{n}\right)}$ such that $P(D)=J$ where $J$ is the square matrix of order $n$ whose all entries are one and $k$ is the unique sum of each rows of $\mathrm{D}$.

Product of graphs defined by Yeh and Gutman in [14] are as follows:

(1) The Cartesian product $G_{1} \times G_{2}$ :

$$
\mathrm{V}\left(G_{1} \times G_{2}\right)=\mathrm{V}\left(G_{1}\right) \times \mathrm{V}\left(G_{2}\right)
$$

the vertices $u=\left(u_{1}, u_{2}\right)$ and $v=\left(v_{1}, v_{2}\right)$ of $G_{1} \times G_{2}$ are adjacent if and only

if either $\left[u_{1}=v_{1},\left(u_{2}, v_{2}\right) \in \mathrm{E}\left(G_{2}\right)\right]$ or $\left[u_{2}=v_{2},\left(u_{1}, v_{1}\right) \in \mathrm{E}\left(G_{1}\right)\right]$.

(2) The composition (lexicographic product) $G_{l}\left[G_{2}\right]$ :

$$
\mathrm{V}\left(G_{1}\left[G_{2}\right]\right)=\mathrm{V}\left(G_{1}\right) \times \mathrm{V}\left(G_{2}\right)
$$

the vertices $u=\left(u_{1}, u_{2}\right)$ and $v=\left(v_{1}, v_{2}\right)$ of $G_{1}\left[G_{2}\right]$ are adjacent if and only if either $\left[u_{1}=v_{1},\left(u_{2}, v_{2}\right) \in \mathrm{E}\left(G_{2}\right)\right]$ or $\left[\left(u_{1}, v_{1}\right) \in \mathrm{E}\left(G_{1}\right)\right]$.

(3) The Kronecker (tensor) product $G_{1} \otimes G_{2}$ :

$$
\mathrm{V}\left(G_{1} \otimes G_{2}\right)=\mathrm{V}\left(G_{1}\right) \times \mathrm{V}\left(G_{2}\right) ;
$$

the vertices $u=\left(u_{1}, u_{2}\right)$ and $v=\left(v_{1}, v_{2}\right)$ of $G_{1} \otimes G_{2}$ are adjacent if and only if $\left[\left(u_{1}, v_{1}\right)\right.$ $\left.\in \mathrm{E}\left(G_{1}\right)\right]$ and $\left[\left(u_{2}, v_{2}\right) \in \mathrm{E}\left(G_{2}\right)\right]$.

(4) The join $G_{1} \nabla G_{2}$ :

$$
\begin{aligned}
& \mathrm{V}\left(G_{1} \nabla G_{2}\right)=\mathrm{V}\left(G_{1}\right) \cup \mathrm{V}\left(G_{2}\right) ; \\
& \mathrm{E}\left(G_{1} \nabla G_{2}\right)=\mathrm{E}\left(G_{1}\right) \cup \mathrm{E}\left(G_{2}\right) \cup\left\{\left(u_{1}, u_{2}\right) \mid u_{1} \in \mathrm{V}\left(G_{1}\right), u_{2} \in \mathrm{V}\left(G_{2}\right)\right\}
\end{aligned}
$$

\section{VDC-spectra of some graphs}

In this section we find the $V D C$-spectrum of join of two graphs, cartesian product of an arbitrary graph with $K_{2}$, double graph, lexicographic product of a graph with $K_{2}$, double odd graph and extended double cover graph.

\subsection{Join of two graphs $G_{1} \nabla G_{2}$}

Theorem 3.1. For $i=1,2$ let $G_{i}$ be a $r_{i}$ regular graph on $n_{i}$ vertices with adjacency spectrum $\lambda_{1}\left(\mathrm{G}_{\mathrm{i}}\right) \geq \lambda_{2}\left(\mathrm{G}_{\mathbf{i}}\right) \geq \ldots \geq \lambda_{\mathrm{n}}\left(\mathrm{G}_{\mathbf{i}}\right)$ and diameter 2 then the $V D C$ - spectrum of $G_{1} \nabla G_{2}$ consists of $\lambda_{i}\left(G_{1}\right)-n+2$ for $i=2,3 \ldots, n_{l}$, 
Renny P Varghese and Susha D.

$\lambda_{j}\left(G_{2}\right)-n+2$ for $j=2,3, \ldots, n_{2}$ and two roots of the equation;

$x^{2}-\left((n-2)^{2}+r_{1}+r_{2}\right) x+\left((n-2)\left(n_{1}-1\right)+r_{1}\right)\left((n-2)\left(n_{2}-1\right)+r_{2}\right)$

$-n_{1} n_{2}(n-1)^{2}=0$. where $n=n_{1}+n_{2}$.

Proof: From the definition of join of two graphs, the distance matrix of the join $G_{1} \nabla G_{2}$ is

$$
\mathrm{D}\left(G_{1} \nabla G_{2}\right)=\left[\begin{array}{lr}
D_{1} & J_{n_{1 \times n_{2}}} \\
J_{n_{2 \times n_{1}}} & D_{2}
\end{array}\right],
$$

where $D_{1}$ and $D_{2}$ are the distance matrices of $G_{1}$ and $G_{2}$ respectively and $J$ is the matrix of all entries equal to 1 .

The $V D C$ - matrix of $G_{1} \nabla G_{2}$,

where $n=n_{1}+n_{2}$.

$$
\mathrm{B}=\left[\begin{array}{lr}
n(J-I)_{n_{1}} & n J_{n_{1 \times n_{2}}} \\
n J_{n_{2 \times n_{1}}} & n(J-I)_{n_{2}}
\end{array}\right]-\left[\begin{array}{lr}
D_{1} & J_{n_{1 \times n_{2}}} \\
J_{n_{2 \times n_{1}}} & D_{2}
\end{array}\right],
$$

Since $\operatorname{diam}(G)=2$, the distance matrix $D=A(G)+2 \overline{A(G)}=2(J-I)-A(G)$

$$
\mathrm{B}=\left[\begin{array}{lr}
(n-2)(J-I)_{n_{1}}+A\left(G_{1}\right) & (n-1) J_{n_{1 \times n_{2}}} \\
(n-1) J_{n_{2 \times n_{1}}} & (n-2)(J-I)_{n_{2}}+A\left(G_{2}\right)
\end{array}\right] .
$$

Since $G_{1}$ is $r_{1}$ - regular, it has an eigenvector $\mathbf{1}_{n_{1}}$, a vector with all entries equal to 1 , corresponding to the eigenvalue $r_{1}$. All other eigenvectors are orthogonal to $\mathbf{1}_{n_{1}}$. Let $\lambda\left(G_{1}\right)$ be an eigenvalue of the adjacency matrix $A\left(G_{1}\right)$ of $G_{1}$ with eigenvector $X$ such that $1^{T} X=0$. Then $(X, 0)^{T}$ is an eigenvector corresponding to the eigenvalue $-(n-2)+\lambda\left(G_{1}\right)$. This is because

$$
\begin{aligned}
{\left[\begin{array}{l}
(n-2)(J-I)_{n_{1}}+A\left(G_{1}\right) \\
(n-1) J_{n_{2 \times n_{1}}}
\end{array}\right.} & (n-1) J_{n_{1 \times n_{2}}} \\
& =\left[\begin{array}{c}
(-(n-2)+\lambda \\
X \\
0
\end{array}\right] \\
& =\left(\lambda\left(G_{1}\right)-n+2\right)\left[\begin{array}{l}
X \\
0
\end{array}\right] .
\end{aligned}
$$

Similarly $(0, Y)^{T}$ is also an eigenvector corresponding to $\lambda\left(G_{2}\right)$. In an equivalent manner we can prove $-(n-2)+\lambda\left(G_{2}\right)$ is an eigenvalue of $A\left(G_{2}\right)$ and the corresponding eigenvectors are $(0, Y)^{T}$.

In this way we obtain eigenvectors of the form $(X, 0)^{T}$ and $(0, Y)^{T}$ all orthogonal to $\left(\mathbf{1}_{\mathrm{n} 1}, 0\right)^{\mathrm{T}}$ and $\left(0, \mathbf{1}_{\mathrm{n} 2}\right)^{\mathrm{T}}$. Thus we obtain $n_{1}-1+n_{2}-1=n_{1}+n_{2}-2$ eigenvalues of $\boldsymbol{B}$.

The remaining two vectors of $B$ are of the form $(\alpha \mathbf{1}, \beta \mathbf{1})$ for $(\alpha, \beta) \neq 0$.

Let $x$ be the eigenvalue of B with eigenvector $\tau$.

Then from $\mathrm{B} \tau=x \tau$ we get

$$
\begin{gathered}
(n-2)\left(n_{1}-1\right) \alpha+r_{1} \alpha+(n-1) n_{2} \beta=x \alpha \\
n_{1}(n-1) \alpha+(n-2)\left(n_{2}-1\right) \beta+r_{2} \beta=x \beta
\end{gathered}
$$

By solving equations (1) and (2) we get the remaining two eigenvalues. 


\section{Vertex Distance Complement Spectra of Some Graphs}

\subsection{Cartesian product $G \times K_{2}$}

Theorem 3.2. Let $\mathrm{G}$ be a distance regular graph with distance spectrum

$\left\{\eta_{1}, \eta_{2}, \ldots, \eta_{n}\right\}$ and distance regularity k. Then the $V D C$ - spectrum of $G \times \mathrm{K}_{2}$ is $\operatorname{VDCSpec}\left(G \times \mathrm{K}_{2}\right)=\left(\begin{array}{cccc}4 n^{2}-3 n-2 k & -2\left(n+\eta_{i}\right) & -n & -2 n \\ 1 & 1 & 1 & n-1\end{array}\right)$,

for $i=2,3, \ldots, n$.

Proof: From the definition of Cartesian product, we have distance matrix of $G \times \mathrm{K}_{2}$ i s

$V D C$ matrix of $\mathrm{G} \times \mathrm{K}_{2}$ is

$$
\mathrm{D}\left(G \times \mathrm{K}_{2}\right)=\left[\begin{array}{cc}
D & D+J \\
D+J & D
\end{array}\right]
$$

$$
\begin{gathered}
V D C\left({\left.\mathrm{G} \times \mathrm{K}_{2}\right)}^{V}=\left[\begin{array}{cc}
2 n(J-I) & 2 n J \\
2 n J & 2 n(J-I)
\end{array}\right]-\left[\begin{array}{cc}
D & D+J \\
D+J & D
\end{array}\right]\right. \\
=\left[\begin{array}{cc}
2 n(J-I)-D & (2 n-1) J-D \\
(2 n-1) J-D & 2 n(J-I)-D
\end{array}\right] .
\end{gathered}
$$

By Lemma 2.4 the eigenvalues of $G \times K_{2}$ are those of $2 n(J-I)-D+(2 n-1) J-D$ and $2 n(J-I)-D-(2 n-1) J+D$.

i.e. the eigenvalues of $(4 n-1) J-2 n I-2 D$ and $J-2 n I$.

Using Theorem 2.5 we get the required spectrum.

Corollary 3.3. If $G$ is a $r$ - regular graph with diameter 2 and adjacency spectrum $\left\{r=\lambda_{1}, \lambda_{2}, \ldots, \lambda_{n}\right\}$. Then the $V D C$ - spectrum of $G \times \mathrm{K}_{2}$ is

$\operatorname{VDCSpec}\left(G \times K_{2}\right)$

$=\left(\begin{array}{ccrc}4 n^{2}-7 n-2 r+4 & 2\left(\lambda_{i}+2-n\right) & -n & -2 n \\ 1 & 1 & 1 & n-1\end{array}\right)$,

for $i=2,3, \ldots, n$.

\subsection{Double Graph $D_{2}(G)$}

Definition 3.1. [7] Let $G$ be a graph with vertex set $\mathrm{V}(G)=\left\{v_{1}, v_{2}, \ldots, v_{n}\right\}$. Take another copy of $G$ with the vertices denoted by $\left\{u_{1}, u_{2}, \ldots, u_{n}\right\}$ where $u_{i}$ corresponds to $v_{i}$ for each $i$. Make $u_{i}$ adjacent to all the vertices in $\mathrm{N}\left(v_{i}\right)$, the neighborhood of $v_{i}$, in $G$ for each $i$. The resulting graph is called the double graph of $G$ and is denoted by $D_{2}(G)$.

Theorem 3.4. Let $\mathrm{G}$ be a distance regular graph with distance spectrum $\left\{\eta_{1}, \eta_{2}, \ldots, \eta_{n}\right\}$ and distance regularity $k$. Then the $V D C$ - spectrum of $D_{2}(G)$ is $\operatorname{VDCSpec}\left(D_{2}(G)\right)=\left(\begin{array}{ccc}4 n^{2}-2 n-2-2 k & -2\left(n+1+\eta_{i}\right) & -2(n-1) \\ 1 & 1 & n\end{array}\right)$, for $i=2,3, \ldots, n$.

Proof: From the Definition 3.1, the distance matrix of $D_{2}(G)$ is

$V D C$ matrix of $D_{2}(G)$ is

$$
\mathrm{D}\left(D_{2}(G)\right)=\left[\begin{array}{cc}
D & D+2 I \\
D+2 I & D
\end{array}\right]
$$

$$
\operatorname{VDC}\left(D_{2}(G)\right)=\left[\begin{array}{lr}
2 n(J-I)-D & 2 n J-D-2 I \\
2 n J-D-2 I & 2 n(J-I)-D
\end{array}\right] .
$$


Renny P Varghese and Susha D.

By Lemma 2.4 the eigenvalues of $D_{2}(G)$ are those of $2 n(J-I)-D+2 n J-D-2 I$ together with $2 n(J-I)-D-2 n J+D+2 I$.

ie the eigenvalues of $4 n J-2(n+1) I-2 D$ and $-2(n-1) I$.

Hence the theorem follows using Theorem 2.5.

Corollary 3.5. If $G$ is an $r$ - regular graph with diameter 2 and adjacency spectrum $\left\{r=\lambda_{1}, \lambda_{2}, \ldots, \lambda_{n}\right\}$. Then the $V D C$ - spectrum of $D_{2}(G)$ is

$\operatorname{VDCSpec}\left(D_{2}(\mathrm{G})\right)=\left(\begin{array}{ccc}4 n^{2}-6 n+2 r+2 & -2\left(\lambda_{i}-n+2\right) & -2(n-1) \\ 1 & 1 & n\end{array}\right)$,

for $i=2,3, \ldots, n$.

\subsection{Lexicographic product of $\mathbf{G}$ with $K_{2}$}

Theorem 3.6. Let $G$ be a distance regular graph with distance spectrum $\left\{\eta_{1}, \eta_{2}, \ldots\right.$, $\left.\eta_{n}\right\}$ and distance regularity $k$. Then the VDC - spectrum of the lexicographic product of $G$ with $\mathrm{K}_{2}, G\left[\mathrm{~K}_{2}\right]$, is

$$
\operatorname{VDCSpec}\left(G\left[\mathrm{~K}_{2}\right]\right)=\left(\begin{array}{ccc}
4 n^{2}-2 n-1-2 k & -\left(2 n+1+2 \eta_{i}\right) & -(2 n-1) \\
1 & 1 & n
\end{array}\right),
$$

for $i=2,3, \ldots, n$.

Proof: From the definition of lexicographic product, we have the distance matrix of $G\left[K_{2}\right]$ is

$V D C$ matrix of $G\left[\mathrm{~K}_{2}\right]$ is

$$
\mathrm{D}\left(G\left[\mathrm{~K}_{2}\right]\right)=\left[\begin{array}{cc}
D & D+I \\
D+I & D
\end{array}\right]
$$

$$
V D C\left(G\left[K_{2}\right]\right)=\left[\begin{array}{lr}
2 n(J-I)-D & 2 n J-D-I \\
2 n J-D-I & 2 n(J-I)-D
\end{array}\right]
$$

By Lemma 2.4 the eigenvalues of $G\left[K_{2}\right]$ are those of $4 n J-(2 n+1) I-2 D$ and $-(2 n-1) I$. Remaining proof follows from Theorem 2.5 .

Corollary 3.7. If $G$ is a $r$ - regular graph with diameter 2 and adjacency spectrum $\left\{r=\lambda_{1}, \lambda_{2}, \ldots, \lambda_{n}\right\}$. Then the $V D C$ - spectrum of $\mathrm{G}\left[\mathrm{K}_{2}\right]$ is

for $i=2,3, \ldots, n$.

$$
\operatorname{VDCSpec}\left(G\left[\mathrm{~K}_{2}\right]\right)=\left(\begin{array}{ccc}
4 n^{2}-6 n+2 r+3 & 2 \lambda_{i}-2 n+3 & -(2 n-1) \\
1 & 1 & n
\end{array}\right),
$$

\subsection{Double odd graph, DO(r)}

Let $n$ and $r$ be two fixed integers. Consider the collection of integers $\mathrm{S}=\{1,2,3, \ldots, \mathrm{n}\}$ and $\left(\begin{array}{l}n \\ r\end{array}\right)$ denote the number of $r$-subsets of S. The graph $J(n ; r ; i)$ with fixed integers $n$, $r$ and $i$ is defined on the vertex set $\left(\begin{array}{l}n \\ r\end{array}\right)$ such that two vertices $\mathrm{T}_{1}$ and $\mathrm{T}_{2}$ are adjacent iff $\left|\mathrm{T}_{1} \cap \mathrm{T}_{2}\right|=\mathrm{r}-\mathrm{i}$.

For $i=1$ the graph $J(n ; r ; 1)=J(n, r)$ is called the Johnson graph. The Kneser graph $\mathrm{K}(\mathrm{n}, \mathrm{r})$ is the Johnson graph $J(n ; r ; r)$ and the odd graph $\mathrm{O}(\mathrm{r})=\mathrm{K}(2 \mathrm{r}+1, \mathrm{r})$. A Double odd graph DO(r) is a graph whose vertices are $r$ - element or $(\mathrm{r}+1)$ element subset of $\{1,2, \ldots, 2 \mathrm{r}+1\}$. Two vertices $\mathrm{T}_{1}$ and $\mathrm{T}_{2}$ are adjacent iff $\mathrm{T}_{1} \subset \mathrm{T}_{2}$ or 


\section{Vertex Distance Complement Spectra of Some Graphs}

$\mathrm{T}_{2} \subset \mathrm{T}_{1}$. Also, the Double odd graph can be constructed as the Kronecker product of $\mathrm{O}(\mathrm{r})$ with the path $\mathrm{P}_{2}$.

For more details see [1].

Theorem 3.8. [3] The distance spectrum of Johnson graph $J(n, r)$ is given by

$\operatorname{Spec}_{D}(J(n, r))=\left(\begin{array}{ccc}s(n, r) & 0 & \frac{-s(n, r)}{n-1} \\ 1 & \left(\begin{array}{l}n \\ r\end{array}\right)-n & n-1\end{array}\right)$,

where, $s(n, r)=\sum_{j=0}^{r} j\left(\begin{array}{l}r \\ j\end{array}\right)\left(\begin{array}{c}n-r \\ j\end{array}\right)$.

Theorem 3.9. [1] Let $J(2 r+1, r)$ be the Johnson graph of order $\mathrm{N}=\left(\begin{array}{c}2 \mathrm{r}+1 \\ \mathrm{r} \\ \mathrm{r}\end{array}\right)$. Let

$\mathrm{r} \geq 2$, the distance spectrum of the Double odd graph DO(r) is

$\operatorname{Spec}_{D}(D O(r))=$

$\left(\begin{array}{cccc}(2 r+1) N & 0 & \frac{-s(2 r+1, r)}{n-1} & -(2 r+1) N+4 s(2 r+1, r) \\ 1 & 2 N-2 r-2 & 2 r & 1\end{array}\right)$, where, $s(n, r)=\sum_{j=0}^{r} j\left(\begin{array}{l}r \\ j\end{array}\right)\left(\begin{array}{c}n-r \\ j\end{array}\right)$.

Theorem 3.10. Let $\mathrm{G}$ be an arbitrary graph with distance spectrum $\left\{\eta_{1}, \eta_{2}, \ldots, \eta_{n}\right\}$. Then the $V D C$ - spectrum of the double odd graph, $\mathrm{DO}(\mathrm{r})$, is $\operatorname{VDCSpec}(D O(r))=$

$$
\left(\begin{array}{cccc}
N(4 N-2 r-3) & -2 N & (2 r-1) N-4 s & -\left(2 N-\frac{2 s}{r}\right) \\
1 & 2(N-r-1) & 1 & 2 r
\end{array}\right),
$$

where, $\mathrm{N}=\left(\begin{array}{c}2 \mathrm{r}+1 \\ \mathrm{r}\end{array}\right)$ and $s=s(2 r+1, r)=\sum_{j=0}^{r} j\left(\begin{array}{l}r \\ j\end{array}\right)\left(\begin{array}{c}r+1 \\ j\end{array}\right)$.

Proof: We have from Theorem 3.8 distance matrix of $D O(r)$ is

$$
\left[\begin{array}{lr}
2 D & (2 r+1) J-2 D \\
(2 r+1) J-2 D & 2 D
\end{array}\right]
$$

where $D$ is the distance matrix of the Johnson graph $J(2 r+1, r)$ and $J$ in the above matrix is a square matrix of order $2 r+1$ having all entries equal to 1 .

Hence the $V D C$ matrix of $\mathrm{DO}(\mathrm{r})$ is

$$
V D C(D O(r))=\left[\begin{array}{lr}
2 N(J-I)-2 D & (2 N-2 r-1) J+2 D \\
(2 N-2 r-1) J+2 D & 2 N(J-I)-2 D
\end{array}\right] .
$$

By Lemma 2.4 the $V D C$-eigenvalues of $D O(r)$ are those of $(4 N-2 r-1) J-2 N I$ and $(2 r+1) J-2 N I-4 D$.

By Theorem 2.5 the eigenvalues corresponding to $(4 N-2 r-1) J-2 N I$ are

$$
\left(\begin{array}{cc}
(4 N-2 r-3) N & -2 N \\
1 & N-1
\end{array}\right) \text {. }
$$

Since $D$ is the distance matrix of the Johnson graph $J(2 r+1, r)$, from Theorem 3.8, 
Renny P Varghese and Susha D.

$\operatorname{Spec}_{D}(J)=\left(\begin{array}{ccc}s(2 r+1, r) & 0 & \frac{-s(2 r+1, r)}{2 r} \\ 1 & N-(2 r+1) & 2 r\end{array}\right)$,

where, $\mathrm{N}=\left(\begin{array}{c}2 \mathrm{r}+1 \\ \mathrm{r}\end{array}\right)$ and $\mathrm{s}=s(2 r+1, r)=\sum_{j=0}^{r} j\left(\begin{array}{l}r \\ j\end{array}\right)\left(\begin{array}{c}n-r \\ j\end{array}\right)$.

Using Theorem 3.9 and Equation (4), the eigenvalues corresponding to $(2 r+1) J-2 N I-4 D$ is

$$
\left(\begin{array}{ccc}
(2 r-1) N-4 s(2 r+1, r) & -2 N & -\left(2 N-\frac{2 s(2 r+1, r)}{r}\right) \\
1 & N-(2 r+1) & 2 r
\end{array}\right)
$$

Combining Equations (3) and (5) we get the required $V D C$ - spectrum of $\mathrm{DO}(\mathrm{r})$.

\subsection{Extended double cover graph}

The extended double cover of a graph was first introduced in 1986 by Alon [2] in connection with the study of networks.

Definition 3.2. [2] Let $G$ be a simple graph with vertex set $\mathrm{V}(G)=\left\{v_{1}, v_{2}, \ldots, v_{n}\right\}$. The extended double cover of $G$, denoted by $\mathrm{D}^{*}(\mathrm{G})$, is the bipartite graph with bipartition $(\mathrm{X}, \mathrm{Y})$, where $\mathrm{X}=\left\{x_{1}, x_{2}, \ldots, x_{n}\right\}$ and $\mathrm{Y}=\left\{y_{1}, y_{2}, \ldots, y_{n}\right\}$, in which $x_{i}$ and $y_{i}$ are adjacent if and only if $i=j$ or $v_{i}$ and $v_{j}$ are adjacent in $G$.

Theorem 3.11. If $G$ is a $r$-regular graph on $n$ vertices with diameter 2 and adjacency spectrum $\left\{r=\lambda_{1}, \lambda_{2}, \ldots, \lambda_{n}\right\}$. The $V D C$ - spectrum of $\mathrm{G}^{*}$, the extended double cover of $\mathrm{G}$ is $\operatorname{VDCSpec}\left(\mathrm{G}^{*}\right)=$

$\left(\begin{array}{cccc}4 n^{2}-7 n+2 r+4 & -(n+2 r) & 2 \lambda_{i}-2 n+4 & -2\left(n+\lambda_{i}\right. \\ 1 & 1 & 1 & 1\end{array}\right)$

for $i=2,3, \ldots, n$.

Proof: Let A be the adjacency matrix of $G$. Since $\operatorname{diam}(G)=2$, we have $D(G)=$ $A(G)+2 \overline{A(G)}$. Then by the definition of $G^{*}$, the distance matrix of $G^{*}$ can be written as,

$V D C$ matrix of $\mathrm{G}^{*}$ is

$$
\mathrm{D}\left(\mathrm{G}^{*}\right)=\left[\begin{array}{lr}
2(J-I) & 3 J-2 I-2 A \\
3 J-2 I-2 A & 2(J-I)
\end{array}\right] .
$$

$$
\operatorname{VDC}\left(\mathrm{G}^{*}\right)=\left[\begin{array}{lr}
(2 n-2)(J-I) & (2 n-3) J+2 I+2 A \\
(2 n-3) J+2 I+2 A & (2 n-2)(J-I)
\end{array}\right] .
$$

By Theorem 3.5, the eigenvalues of $G^{*}$ are those of $(2 n-2)(J-I)+(2 n-3) J+$ $2 I+2 A=(4 n-5) J+(4-2 n) I+2 A$ and $(2 n-2)(J-I)-(2 n-3) J-2 I-2 A$ $=J-2 n I-2 A$.

Using Theorem 2.5 we get the required result.

Theorem 3.12. Let $G$ be an A - integral graph with diameter atmost 2 , then the following class of graphs are $V D C$ - integral.

(1) Cartesian product of $\mathrm{G}$ with $\mathrm{K}_{2}: G \times \mathrm{K}_{2}$

(2) Double graph: $\mathrm{D}_{2}(G)$ 


\section{Vertex Distance Complement Spectra of Some Graphs}

(3) Lexicographic product of $G$ with $\mathrm{K}_{2}: G\left[\mathrm{~K}_{2}\right]$

(4) Extended double cover graph: $\mathrm{G}^{*}$

Proof: From Corollaries 3.3, 3.5, 3.7 and Theorem 3.11, it is clear that if $G$ is A-integral then the $V D C$ - spectrum of $G \times K_{2}, D_{2}(G), G\left[K_{2}\right]$ and $\mathrm{G}^{*}$ consists only of integers.

\section{4. $V D C$-energy}

Energy of the graph $\mathrm{G}$ is introduced by Gutman [9]. Research is going on this field and many results obtained in this regard. For more details see [9 - 13].

Definition 4.1. [12] $V D C$ - energy is the sum of the absolute values of the eigenvalues of the vertex distance complement matrix. It is denoted by $\operatorname{VDC} E(G)$. If $\left\{\theta_{1}, \theta_{2}, \ldots, \theta_{n}\right\}$ are the $V D C$ - spectrum of a graph $\mathrm{G}$ then the $V D C$ - energy is,

$$
\operatorname{VDCE}(G)=\sum_{i=1}^{n}\left|\theta_{i}\right| \text {. }
$$

Theorem 4.1. Let $\mathrm{G}$ be a connected $\mathrm{r}$ - regular graph on $\mathrm{n}$ vertices with a diameter two. Then $V D C$ - energy of $\mathrm{G} \times \mathrm{K}_{2}$ is

(i) The second largest eigenvalue of $\mathrm{G}$ is less than $n-2$ then,

$$
\operatorname{VDCE}\left(G \times K_{2}\right)=2\left(4 n^{2}-7 n+2 r+4\right)
$$

(ii) The smallest adjacency eigenvalue of $\mathrm{G}$ is greater than or equal to $n-2$ then,

$$
\operatorname{VDCE}\left(G \times K_{2}\right)=2 n(2 n-1)
$$

Proof: Since $G$ is $r$-regular, by Theorem $2.54 n^{2}-7 n-2 r+4$, the largest $V D C$ eigenvalue of $G \times K_{2}$, and is positive.

(i) By assumption $\lambda_{i}+2-n<0$, for $i=2,3, \ldots, n$.

Hence from Corollary 3.3 it is clear that $4 n^{2}-7 n+2 r+4$ is the only positive $V D C$ eigenvalue of $G \times K_{2}$. Since the diagonal entries of $V D C(G)$ are zero, algebraic sum of the $V D C$ - eigenvalues of any graph is zero. Therefore,

$$
\begin{aligned}
\operatorname{VDCE}\left(G \times K_{2}\right) & =2 \times\left(4 n^{2}-7 n+2 r+4\right) \\
& =2\left(4 n^{2}-7 n+2 r+4\right) .
\end{aligned}
$$

(ii) If $\lambda_{i}+2-n \geq 0$ then from Corollary $3.3,-n$ and $-2 n$ are the only negative eigenvalue of $V D C\left(G \times K_{2}\right)$ which repeats 1 and $n-1$ times respectively.

$$
\begin{aligned}
\operatorname{VDCE}\left(G \times K_{2}\right) & =2[n \times 1+2 n \times(n-1)] \\
& =2 n(2 n-1) .
\end{aligned}
$$

Theorem 4.2. Let $G$ be a connected $r$ - regular graph on $n>2$ vertices with diameter

2. Then $V D C$ - energy of the double graph $D_{2}(G)$ is

(i) The second largest eigenvalue of $\mathrm{G}$ is less than $n-2$ then,

$$
\operatorname{VDCE}\left(D_{2}(G)\right)=2\left(4 n^{2}-6 n+2 r+2\right) \text {. }
$$

(ii)The smallest adjacency eigenvalue of $G$ is greater than or equal to $n-2$ then,

$$
\operatorname{VDCE}\left(D_{2}(G)\right)=2 n(2 n-1) \text {. }
$$

Proof: We have $\left(4 n^{2}-6 n+2 r+2\right)$ is the largest $V D C$ - eigenvalue of $D_{2}(G)$. By Theorem 2.5 , it is positive. 
Renny P Varghese and Susha D.

(i) By assumption $\lambda_{i}-n+2<0$ for all $i=2,3, \ldots, n$.

Hence from Corollary 3.5 it is clear that $-2(n-1)$ is the only negative $V D C$ eigenvalues of $D_{2}(G)$ which repeats $n$ times. Therefore,

$$
\begin{aligned}
& \operatorname{VDCE}\left(D_{2}(G)\right)=2 \times[n \times 2(n-1)] \\
= & 4 n(n-1) .
\end{aligned}
$$

(ii) If $\lambda_{i}-n+2 \geq 0$, from Corollary $3.5,4 n^{2}-6 n+2 r+2$ is the only positive VDC eigenvalue of $D_{2}(G)$. Therefore,

$$
\begin{aligned}
\operatorname{VDCE}\left(D_{2}(G)\right) & =2 \times\left(4 n^{2}-6 n+2 r+2\right) \\
& =4\left(2 n^{2}-3 n+r+1\right) .
\end{aligned}
$$

Theorem 4.3. Let $G$ be a connected $r$ - regular graph on $n$ vertices with diameter 2 . Then $V D C$ - energy of the lexicographic product of $G$ with $\mathrm{K}_{2}, \mathrm{G}\left[\mathrm{K}_{2}\right]$, is

(i) The second largest eigenvalue of $G$ is less than $\frac{2 n-3}{2}$ then,

$$
\operatorname{VDCE}\left(G\left[K_{2}\right]\right)=2\left[4 n^{2}-6 n+2 r+3\right] .
$$

(ii) The smallest adjacency eigenvalue of $G$ is greater than or equal to $\frac{2 n-3}{2}$ then,

$$
\operatorname{VDCE}\left(G\left[K_{2}\right]\right)=2 n(2 n-1) \text {. }
$$

Proof: The proof of the Theorem is similar in lines that of the above Theorem.

Theorem 4.4. Let $G$ be a connected $\mathrm{r}$ - regular graph with $\mathrm{n}>4$ vertices. Then $V D C$ energy of $\mathrm{G}^{*}$, the extended double cover of $G$, is

(i) The second largest eigenvalue of $G$ is less than $n-4$ then,

$$
\operatorname{VDCE}\left(G^{*}\right)=2\left[4 n^{2}-7 n+2 r+4\right] \text {. }
$$

(ii)The smallest adjacency eigenvalue of $G$ is greater than or equal to $n-4$ then,

$$
\operatorname{VDCE}\left(G^{*}\right)=2 n(2 n-1) \text {. }
$$

Proof: Since $G$ is $r$ - regular, $4 n^{2}-7 n-2 r+4$ is positive. Also $-r \leq \lambda_{i} \leq r$ for $i=2,3, \ldots, n$.

So $-n<-r \leq \lambda_{i} \Rightarrow n+\lambda_{i}>0$ for $i=2,3, \ldots, n$.

(i) By assumption $\lambda_{i}+2-n<0$, for $i=2,3, \ldots, n$.

Hence from Theorem 3.11, it is clear that $4 n^{2}-7 n-2 r+4$ is the only positive VDC eigenvalue of $G^{*}$. Since the diagonal entries of $V D C(G)$ are zero, algebraic sum of the $V D C$ - eigenvalues of any graph is zero. Therefore,

$$
\begin{gathered}
\operatorname{VDCE}\left(G^{*}\right)=2 \times\left(4 n^{2}-7 n-2 r+4\right) \\
=2\left(4 n^{2}-7 n+2 r+4\right) .
\end{gathered}
$$

(ii) If $\lambda_{i}+2-n \geq 0$ then from Theorem $3.11-(n+2 r)$ and $-2\left(\lambda_{i}+n\right)$,

$i=2,3, \ldots, n$ are the only negative $V D C$ - eigenvalue of $G^{*}$.

We have $\sum_{i=1}^{n} \lambda_{i}=0 \Rightarrow \sum_{i=2}^{n} \lambda_{i}=-r$,

$\operatorname{VDCE}\left(\mathrm{G}^{*}\right)=2\left[(n+2 r) \times 1+2 \sum_{i=2}^{n}\left(n+\lambda_{i}\right)\right.$

$=2[n+2 r+2((n-1) n-r)]$

$=2 n(2 n-1)$. 


\section{Vertex Distance Complement Spectra of Some Graphs}

Definition 4.2. Two connected graphs $G_{1}$ and $G_{2}$ are said to be vertex distance complement equienergetic or $\operatorname{VDC}$ - equienergetic if $\operatorname{VDCE}\left(G_{1}\right)=\operatorname{VDCE}\left(G_{2}\right)$.

Theorem 4.5. Let $G$ be a $r$ - regular graph with diameter 2, then $\mathrm{G} \times \mathrm{K}_{2}$ and $\mathrm{G}^{*}$ are VDC - equienergetic graphs.

Proof: Proof of the theorem follows from Theorems 4.1 and 4.2.
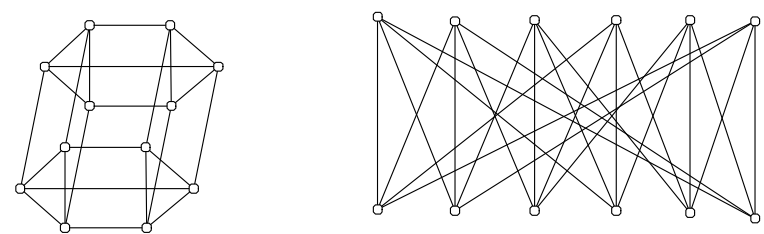

Figure 1: $V D C$-equienergetic graphs with $V D C$ - energy 224.

\section{Conclusion}

The spectral graph theory has various applications in the field of science like theoretical chemistry, quantum mechanics, statistical physics, computer, information science etc. There are mainly two models QSPR and QSAR which are used for the study of molecular design of chemical compound. The vertex distance complement matrix is one of the important sources of structural description for QSPR and QSAR models. In this paper we construct the $V D C$ - spectrum and $V D C$ - energy of some class of graphs. Here we discuss some infinite family of $V D C$ - integral graphs. As an application we can give the $V D C$-energy of $G \times K_{2}, G\left[K_{2}\right], D_{2}(G)$ and the extended double cover graph. Also we proved that the cartesian product $G \times K_{2}$ and the extended double cover graph of $G$ are $V D C$-equienergetic graphs.

Acknowledgements. The authors are thankful to the reviewer for their comments and suggestions for improving the quality of this paper. Also the author thankful to the University Grants Commission of Government of India for providing fellowship under the FDP in the $X I I^{\text {th }}$ plan.

\section{REFERENCES}

1. G.Aalipour et al., On the distance spectra of graphs, Linear Algebra and its Applications, 497 (2016) 66-87.

2. N.Alon, Eigenvalues and expanders, Combinatorica, 6 (1986) 83-96.

3. F.Atik and P.Panigrahi, On the distance spectrum of distance regular graphs, Linear Algrbra and its Applications, 478 (2015) 256-273.

4. D.M.Cvetković, M.Doob and H.Sachs, Spectra of Graphs, Theory and Applications, Third edition, Johann Am-brosius Barth Heidelberg, 1995.

5. R.L.Graham, H.O.Pollak, On the addressing problem for loop switching, Bell System Tech. J., 50 (1971) 2495-2519.

6. G.Indulal and I.Gutman, On the distance spectra of some graphs, Mathematical Communication, 13 (2008) 123-131.

7. G.Indulal and A.Vijayakumar, On a pair of equienergetic graphs, MATCH Commun. Math. Comput. Chem., 55 (2006) 83-90. 
Renny P Varghese and Susha D.

8. D.Jenẽzić, A.Milïcević, S.Nikolić and N.Trinajstić, Graph Theoretical Matrices in Chemistry, Uni. Kragujevac, Kragujevac, 2007.

9. X. L.Li, Y.T.Shi and I.Gutman, Graph Energy, Springer-Verlag New York, Inc., 2012.

10. T.K.Mathew Varkey and J.K.Rajan, On the spectrum and energy of concatenated graphs, Annals of Pure and Applied Mathematics, 14(3) (2017) 555-579.

11. S.Meenakshi and S.Lavanya, A survey on energy of graphs, Annals of Pure and Applied Mathematics, 8(2) (2014) 183-191.

12.R.P.Varghese and D.Susha, Vertex distance complement spectra of regular graphs and its line graphs, Int. J. of Appl. Math. Analysis and Applications, 12(2) (2017) 221-231.

13. D.S.Revankar, M.M.Pattil and H.S.Remane, On eccentricity sum eigenvalues and eccentricity sum energy of a graph, Annals of Pure and Applied Mathematics, 13(1) (2017) 123-130.

14. Y.N.Yeh and I.Gutman, On the sum of all distances in composite graphs, Discrete Math. 135 (1994) 359-365. 